Research paper

\title{
Value of measurement of nuclear diameter of cells in fine needle aspiration smears in the diagnosis of follicular lesions of the thyroid
}

\author{
M.P.G.N.S. Jayawardhana ${ }^{1}$, N.V. Ratnatunga ${ }^{1}$, R.O. Thattil ${ }^{2}$ \\ ${ }^{1}$ Department of Pathology, Faculty of Medicine, University of Peradeniya, Sri Lanka. \\ ${ }^{2}$ Department of Crop Science, Faculty of Agriculture, University of Peradeniya, Sri Lanka.
}

Submitted on 03.05.2021. Accepted for publication on 05.10.2021.

\begin{abstract}
Introduction: Subjective assessment of thyroid epithelial cells in aspiration cytology smears is often not useful in distinguishing benign and malignant thyroid follicular lesions.

Objectives: To determine whether objective measurement of the nuclear diameter of thyroid follicular epithelial cells in smears would be useful in distinguishing benign and malignant thyroid follicular lesions.

Method: Fine needle aspiration cytology of histologically proven follicular adenoma (FA, $\mathrm{n}=50)$, hyperplastic nodule $(\mathrm{HN}, \mathrm{n}=38)$ and follicular carcinoma ( $F C, n=24)$ stained with

two stains ( $p>0.25)$. The one-way ANOVA test showed that there was a significant difference between the mean nuclear diameters of these three groups $(p<0.001)$. The post hoc test showed that there was a significant difference between $F A$ versus $F C$ and $H N$ versus $F C$ $(p<0.001)$. A significant difference was also seen between FA and HN ( $p<0.05)$. The 95\% confidence intervals $(\mathrm{Cl})$ showed that $\mathrm{FC}$ had a distinct range of nuclear diameters which were higher than $\mathrm{FA}$ and $\mathrm{HN}$. However, the $95 \% \mathrm{Cl}$ values for $\mathrm{FA}$ and $\mathrm{HN}$ showed an overlapping grey area.
\end{abstract} Papanicolaou stain and haematoxylin and eosin stain were assessed. The "cellSens" computer software (Version 1.16) was used with an Olympus BX53 microscope for the measurement of nuclear diameters. The nuclear diameters of 100 cells ( $\times 400$ magnification) were measured from each case and the mean and standard deviation were calculated. Statistical analysis was carried out with a two-sample pooled t-test and one-way ANOVA test with post hoc testing.

Results: The two-sampled pooled t-test showed that there was no significant difference in nuclear diameters between the

Corresponding author: Dr Sanjeewani Jayawardhana Department of Pathology, Faculty of Medicine University of Peradeniya, Sri Lanka sanjeewanijayawardhana77@gmail.com

Conclusion: Objective measurement of nuclear diameter in thyroid cytology smears may prove to be useful in distinguishing benign follicular lesions from malignant follicular lesions.

Keywords: follicular lesions, mean nuclear diameter, cytology

\section{Introduction}

Fine needle aspiration cytology is a widely used minimally invasive diagnostic technique in the assessment of thyroid lesions in Sri Lanka and the world over (1). Detection and classification of thyroid follicular lesions based on the nuclear structure in histopathology images were carried out by Wei Wang et al. with promising results (2).

This is an open access article licensed under a Creative Commons Attribution-ShareAlike 4.0 International License. (CC BYSA 4.0), which permits unrestricted use, distribution and reproduction in any medium, provided the original author and source are attributed and materials are shared under the same license. 
To the best of our knowledge, cytomorphometric features to distinguish follicular lesions from each other are poorly defined. We postulated that when a cell becomes hyperplastic or neoplastic, there would be an increase in nuclear size. Other studies have assessed nuclear perimeter, nuclear area and large and small nuclear diameters in thyroid cytomorphometry $(3,4)$. We decided to investigate whether measurement of the nuclear diameter of cells in cytology smears would help to distinguish between FC, FA and HN.

\section{Methods}

The study population comprised fine needle aspiration smears of histologically proven FA, $\mathrm{HN}$ and FC reported from 2012 to 2015 at the teaching hospitals of Peradeniya, Kandy, Anuradhapura and Kurunegala. In order to minimize the effects of sampling error only cases with solitary nodules or samples obtained by ultrasound-guided aspiration were included. All cases fulfilling the inclusion and exclusion criteria were included. Fine needle aspiration cytology smears of 50 cases of FA, 38 cases of $\mathrm{HN}$ and 24 cases of FC were analysed. The cytology smears had been stained by either Papanicolaou (Pap) or haematoxylin and eosin (H\&E) stains. We assumed that the nuclear diameter would not change with the two staining methods and confirmed this with two-sample pooled t-tests for both stains in each of the three diagnostic categories. In each smear, the maximum nuclear diameters of 100 cells were measured. Each nuclear diameter was measured on a high-power microscope field (x400) with 150\% magnification using "cellSens" (Version 1.16) software and a line measurement curser of a branded computer attached to an Olympus BX53 microscope. Measurements were taken by the principal investigator who was blind to the type of follicular lesion. The $150 \%$ magnification was used because it gave the best nuclear demarcation. One hundred cells were selected from well preserved and evenly spread areas of the smear. Crushed, distorted, air-dried and bizarre forms of cells were excluded.

The nuclear diameters were measured in 5000 cells from FAs, 3800 cells from HNs and 2400 cells from FCs. Statistical analysis was performed using IBM SPSS statistics version 22 (2013). The mean and standard deviation of the mean nuclear diameter in each diagnostic category was calculated separately for Pap and H\&E-stained smears. The concordance between the mean nuclear diameters in the Pap and H\&E-stained smears was calculated using the two-sample pooled t-test for each diagnostic category. The one-way ANOVA test was performed to assess the significance of the measurement of mean nuclear diameters to distinguish between FA, $\mathrm{HN}$ and $\mathrm{FC}$. The $95 \% \mathrm{Cl}$ were calculated for each diagnostic category.

\section{Results}

Two-sampled pooled t-tests showed that there was no statistically significant difference between the mean nuclear diameters in Pap and H\&E-stained smears within each diagnostic category $(P>0.25)$. Therefore, smears stained with both stains were included in the study. The means of mean nuclear diameter and standard deviations of each category are given in Table 1.

The box plot shows that the distribution of the mean nuclear diameters of cells of FC was higher and distinctively separate from benign follicular lesions, FA and HN, which showed an overlapping area (Figure 1). The one-way ANOVA test showed that there was a significant difference between the means of the mean nuclear diameters of these three groups $(p<0.001)$. The post hoc test showed that there was a significant difference between $F A$ versus $F C(p<0.001)$, HN versus $F C(p<0.001)$ and $F A$ versus $H N(p<0.05)$.

Calculation of the $95 \% \mathrm{Cl}$ for the mean nuclear diameters showed that $\mathrm{FC}$ had a distinct range 
Table 1: Means of mean nuclear diameters and standard deviations of each diagnostic category

\begin{tabular}{|l|c|c|}
\hline \multicolumn{1}{|c|}{ Category } & $\begin{array}{c}\text { Means of mean } \\
\text { nuclear } \\
\text { diameters }(\mu \mathrm{m})\end{array}$ & $\begin{array}{c}\text { Standard } \\
\text { deviation } \\
(\boldsymbol{\mu m})\end{array}$ \\
\hline Follicular adenoma (50 cases) - 5000 cells & 7.3 & $+/-0.8$ \\
Pap-stained smears (41 cases) - 4100 cells & 7.3 & $+/-0.7$ \\
H\&E-stained smears (09 cases) - 900 cells & 7.2 & $+/-1.0$ \\
\hline Hyperplastic nodules (38cases) - 3800 cells & 7.0 & $+/-0.9$ \\
Pap-stained smears (28 cases) - 2800 cells & 6.9 & $+/-1.0$ \\
H\&E-stained smears (10 cases) - 1000 cells & 7.1 & $+/-0.8$ \\
\hline Follicular carcinomas (24 cases) - 2400 cells & 9.0 & $+/-0.6$ \\
Pap-stained smears (18 cases) - 1800 cells & 9.0 & $+/-0.7$ \\
H\&E-stained smears (06 cases) - 600 cells & 8.8 & $+/-0.5$ \\
\hline
\end{tabular}

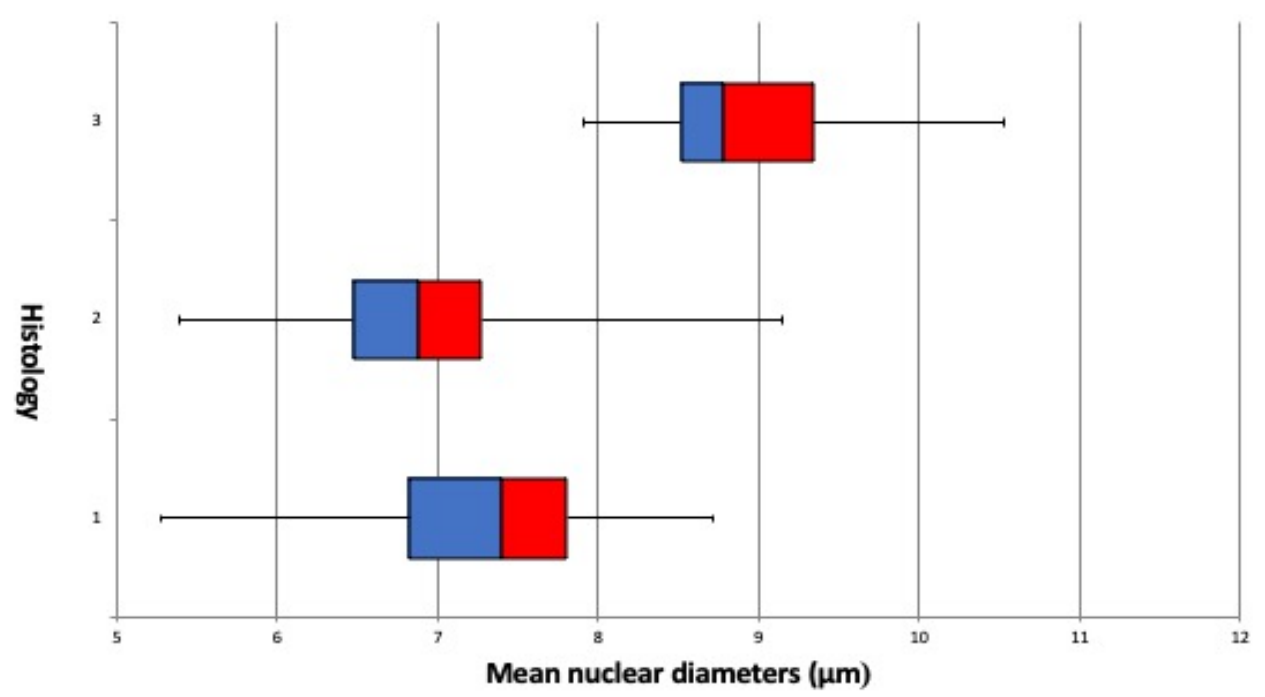

Figure 1: Box plot for the distribution of mean nuclear diameters of thyroid follicular cells in each category. 1 -Follicular adenoma, 2 - Hyperplastic nodule, 3 - Follicular carcinoma

of values (8.7-9.1 $\mu \mathrm{m})$ which were higher than FA (7.1-7.4 $\mu \mathrm{m})$ and HN (6.6-7.2 $\mu \mathrm{m})$. However, the $95 \% \mathrm{Cl}$ values for the mean nuclear diameters of FA (7.1-7.4 $\mu \mathrm{m})$ and HN (6.6-7.2 $\mu \mathrm{m})$ showed an overlapping grey area $(0.1 \mu \mathrm{m})$

\section{Discussion and conclusions}

Previous studies carried out using cytology samples for cytomorphometry to distinguish benign and malignant thyroid follicular lesions have revealed promising results. Features assessed include nuclear diameter, perimeter, nuclear area, nuclear-cytoplasmic ratio, nuclear circular ratio (measured using the ratio of largest to smallest nuclear diameters / L:S ratio) and other parameters such as quantitative assessment of cellular atypia (38). The nuclear-cytoplasmic ratio was higher in malignant cells than benign cells. Another study carried out on H\&E-stained histology samples also showed that nuclear size (area, perimeter, maximum and minimum diameters, nuclear size) and shape ( $L: S$ ratio) were higher in neoplastic lesions (FA, FC and follicular variant of papillary carcinoma) compared to the adenomatous goitre (9).

Our results also show that the measurement of nuclear diameters of thyroid follicular 
epithelial cells on cytology smears could be a useful tool to distinguish between follicular lesions of the thyroid. The difference in nuclear diameters of the three lesions ( $F C, F A, H N)$ is in micrometres and is not discernible by simple light microscopic observation alone. Therefore, objective measurements are mandatory. Validation of this method is required with further studies if this is to be incorporated into the routine practice of thyroid cytology reporting.

The measurement of nuclear diameters in 100 cells during reporting of routine thyroid cytology smears is not practical due to the increased workload upon a pathologist. However, in the future, with the development of computer-assisted software and modern microscopes, this difficulty will not be insurmountable. Studies should also be carried out to determine the minimal number of representative cells that would give accurate values, which would mitigate the increase in the workload. Most importantly, it should be possible to take these measurements using an eyepiece graticule if computer software is not available.

Although there is a statistically significant difference between the mean nuclear diameters of $\mathrm{HN}$ and FA, they cannot be distinguished due to the presence of overlapping values in the calculation of $95 \% \mathrm{Cl}$. However, despite this limitation, the ability to distinguish between benign and malignant thyroid follicular lesions by objective cytological assessment would be of importance to clinicians in the planning of surgeries and other treatment strategies.

\section{References}

1. International Agency for Research in Cancer, WHO. Cancer fact sheets. GLOBOCAN 2012: Estimated cancer incidence, mortality and prevalence worldwide [v 1.0] 2012, Available from: publications.iarc.fr>iarc-Cancerbase. (Accessed 20 $0^{\text {th }}$ February 2016)
2. Wang W, Ozolek JA, and Rohde GK. Detection and classification of thyroid follicular lesions based on nuclear structure from histopathology images. Cytometry Part A. 2010; 77: 485-494.

https://doi.org/10.1002/cyto.a.20853

3. Wright RG, Castles $\mathrm{H}$ and Mortimere RH. Morphometric analysis of thyroid cell aspirates. Journal of Clinical Pathology.1987; 40: 443-5. https://doi.org/10.1136/icp.40.4.443

4. Kirillov V, Emeliyanova O. Differential diagnosis of follicular cancer and follicular adenoma using an expert system based on a set of qualitative signs of cell atypia. Analytical and Quantitative Cytology.2011;33;253-264. PMID: 22611752.

5. Kavantzas N, Tseleni-Balafouta S, Davaris P. Computerized nuclear morphometry and quantitation of angiogenesis in thyroid neoplasms. Journal of Experimental \& Clinical Cancer Research. 2002;21:247-254. PMID: 12148586.

6. Priya SS, Sundaram S. Morphology to morphometry in cytological evaluation of thyroid lesions. Journal of Cytology.2011; 28:98-102.

https://doi.org/10.4103/0970-9371.83462

7. Collins BT, Collins LE. Assessment of malignancy for atypia of undetermined significance in thyroid aspiration biopsy evaluated by whole-slide image analysis. American Journal of Clinical Pathology. 2013;139: 736-745. https://doi.org/10.1309/AJCPQU29GHXYSZRR

8. Nagashima $T$, Suzuki $M$, Oshida $M$, Hashimoto $H$, Yagata $H$, Shishikura $T$ et al. Morphometry in the cytological evaluation of thyroid follicular. Cancer.1998; 84: 1158. PMID: 9570215.

9. Aliad HA, Abdou AG, Bashal M. Computerized nuclear morphometry in the diagnosis of thyroid lesions with predominant follicular pattern. Ecancermedicalscience. 2009; 3:146. https://doi.org/10.3332/ecancer.2009.146 